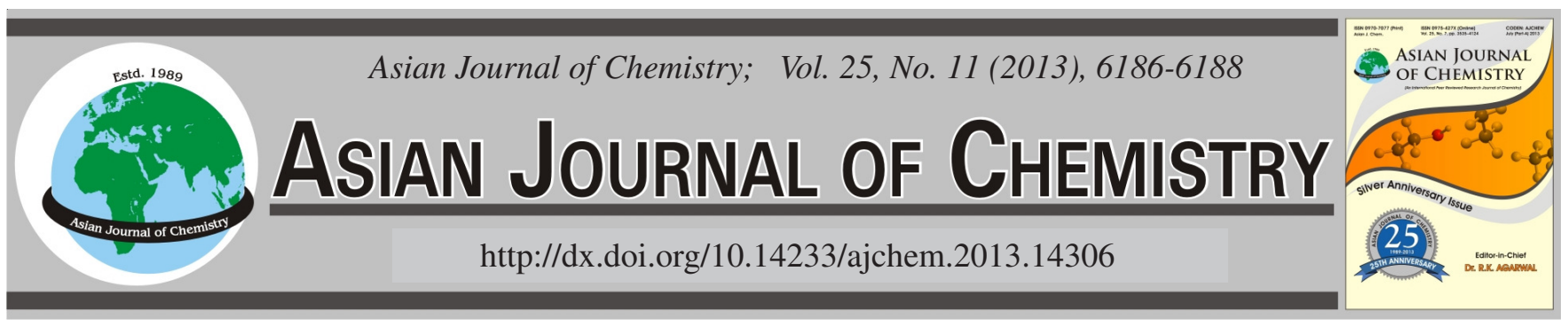

\title{
Supramolecular Structure of 2,4,6-Trinitrophenol
}

Yin-XIA Sun ${ }^{*}$, Zong-Li Ren and Wei-Sheng Meng

School of Chemical and Biological Engineering, Lanzhou Jiaotong University, Lanzhou 730070, P.R. China

*Corresponding author: E-mail: sun_yinxia@163.com

(Received: 13 July 2012;

Accepted: 2 May 2013)

AJC-13431

The compound 2,4,6-trinitrophenol with the molecular formula $\mathrm{C}_{6} \mathrm{H}_{3} \mathrm{~N}_{3} \mathrm{O}_{7}$, crystallizes with two crystallographically independent but chemically identical molecules in the asymmetric unit. The dihedral angle formed by the two benzene rings is about $67.49^{\circ}$. The crystal structure displays four intramolecular hydrogen bonds between the hydrogen atoms from the phenolic alcohols and the oxygen or nitrogen atoms from the nitro groups. In the crystal structure, the intermolecular $\mathrm{O}-\mathrm{H} \cdots \mathrm{O}$ and $\mathrm{C}-\mathrm{H} \cdots \mathrm{O}$ hydrogen bonding interactions link molecules into infinite three-dimensional supramolecular network structure.

Key Words: 2,4,6-Trinitrophenol, Supramolecular structure.

\section{INTRODUCTION}

Nitro compounds, especially aromatic nitro compounds have been widely studied owing to their potential application in, for example, pathology ${ }^{1}$, materials science ${ }^{2}$ and nonlinear optical (NLO) materials ${ }^{3}$. 2,4,6-Trinitrophenol, commonly known as picric acid (TNP), is a nonlinear optical crystal with molecular formula $\mathrm{C}_{6} \mathrm{H}_{3} \mathrm{~N}_{3} \mathrm{O}_{7}$. 2,4,6-Trinitrophenol is also a well-known material used in dyeing industry ${ }^{4}$. Herein, we report the supramolecular structure of 2,4,6-trinitrophenol.

\section{EXPERIMENTAL}

The reagents and solvents were analytical grade reagents used without further purification. $\mathrm{C}, \mathrm{H}$ and $\mathrm{N}$ analyses were carried out with a GmbH VariuoEL V3.00 automatic elemental analyzer. X-Ray single crystal structure was determined on a Bruker Smart APEX CCD area detector. Melting points was measured by the use of a microscopic melting point apparatus made in Beijing Taike Instrument Limited Company and the thermometer was uncorrected.

General procedure: To an acetone solution $(30 \mathrm{~mL})$ of 2,2'-[ethylenedioxybis(nitriloethylidyne)]diphenol (54.0 mg, $0.164 \mathrm{mmol})$ was added an acetone solution $(20 \mathrm{~mL})$ of copper picrate tetrahydrate $(96.0 \mathrm{mg}, 0.162 \mathrm{mmol})$. The colour of the mixed solution turned to brilliant yellow immediately. Then the mixture was placed in a hexane atmosphere, after 2 months, several yellow primatical crystals of the title compound suitable for X-ray diffraction analysis were obtained unexpectedly. Yield, 25.9\%. m.p. 401-402 K. Anal. calcd. (\%) for $\mathrm{C}_{6} \mathrm{H}_{3} \mathrm{~N}_{3} \mathrm{O}_{7}$ :
C, 31.45; H, 1.32; N, 18.34. Found (\%): C, 31.32; H, 1.34; N, 18.27 .

\begin{tabular}{|c|c|}
\hline \multicolumn{2}{|c|}{$\begin{array}{c}\text { TABLE-1 } \\
\text { CRYSTAL DATA AND REFINEMENT } \\
\text { PARAMETERS FOR THE TITLE COMPOUND }\end{array}$} \\
\hline Empirical formula & $\mathrm{C}_{6} \mathrm{H}_{3} \mathrm{~N}_{3} \mathrm{O}_{7}$ \\
\hline Formula weight & 229.11 \\
\hline Temperature & 298(2) K \\
\hline Wavelength & $0.71073 \AA$ \\
\hline Crystal system & Orthorhombic \\
\hline Space group & Pca2(1) \\
\hline Cell dimensions & $\begin{array}{l}a=9.2596(12) \AA, b=19.138(2) \AA, c= \\
9.7075(14) \AA, \alpha=90^{\circ}, \beta=90^{\circ}, \gamma=90^{\circ}\end{array}$ \\
\hline Volume & $1720.3(4) \AA^{3}$ \\
\hline $\mathrm{Z}$ & 8 \\
\hline Density (calculated) & $1.769 \mathrm{mg} / \mathrm{m}^{3}$ \\
\hline Absorption coefficient & $0.166 \mathrm{~mm}^{-1}$ \\
\hline$F_{(000)}$ & 928 \\
\hline Index ranges & $-10 \leq \mathrm{h} \leq 11,-14 \leq \mathrm{k} \leq 22,-11 \leq 1 \leq 11$ \\
\hline Reflections collected/unique & $7969 / 3008[\mathrm{R}(\mathrm{int})=0.0420]$ \\
\hline Data/restraints/parameters & $3008 / 1 / 289$ \\
\hline Goodness of fit indicator & 1.027 \\
\hline $\mathrm{R}[\mathrm{I}>2 \sigma(\mathrm{I})]$ & $\mathrm{R}_{1}=0.0532, \mathrm{wR}_{2}=0.1355$ \\
\hline Largest diff. peak and hole & 0.463 and -0.221 e $\AA^{-3}$ \\
\hline
\end{tabular}

X-Ray structure determination: The single crystal of the 2,4,6-trinitrophenol, with approximate dimensions of 0.38 $\mathrm{mm} \times 0.27 \mathrm{~mm} \times 0.12 \mathrm{~mm}$ was placed on a Bruker Smart 1000 diffractmeter equipped with Apex CCD area detector. The diffraction data were collected using a graphite monochromated $\mathrm{MoK}_{\alpha}$ radition $(\lambda=0.71073 \AA)$ at $298(2) \mathrm{K}$. 


\begin{tabular}{|c|c|c|c|c|c|}
\hline \multicolumn{6}{|c|}{$\begin{array}{c}\text { TABLE- } 2 \\
\text { SELECTED BOND LENGTHS }(\AA) \text { AND ANGLES }\left({ }^{\circ}\right) \text { FOR THE TITLE COMPOUND }\end{array}$} \\
\hline Bond & Lengths & Bond & Lengths & Bond & Lengths \\
\hline $\mathrm{N}(1)-\mathrm{O}(3)$ & $1.187(6)$ & $\mathrm{N}(4)-\mathrm{O}(10)$ & $1.209(7)$ & $\mathrm{N}(5)-\mathrm{O}(11)$ & $1.213(6)$ \\
\hline $\mathrm{N}(1)-\mathrm{O}(2)$ & $1.204(7)$ & $\mathrm{N}(4)-\mathrm{O}(9)$ & $1.223(7)$ & $\mathrm{N}(5)-\mathrm{O}(12)$ & $1.213(7)$ \\
\hline $\mathrm{N}(1)-\mathrm{C}(2)$ & $1.473(7)$ & $\mathrm{N}(4)-\mathrm{C}(8)$ & $1.464(8)$ & $\mathrm{N}(5)-\mathrm{C}(10)$ & $1.460(6)$ \\
\hline $\mathrm{N}(2)-\mathrm{O}(5)$ & $1.210(6)$ & $\mathrm{N}(3)-\mathrm{O}(6)$ & $1.206(6)$ & $\mathrm{N}(6)-\mathrm{O}(14)$ & $1.173(6)$ \\
\hline $\mathrm{N}(2)-\mathrm{O}(4)$ & $1.232(6)$ & $\mathrm{N}(3)-\mathrm{O}(7)$ & $1.241(6)$ & $\mathrm{N}(6)-\mathrm{O}(13)$ & $1.181(7)$ \\
\hline$N(2)-C(4)$ & $1.474(7)$ & $N(3)-C(6)$ & $1.448(6)$ & $\mathrm{N}(6)-\mathrm{C}(12)$ & $1.477(8)$ \\
\hline $\mathrm{O}(8)-\mathrm{C}(7)$ & $1.368(7)$ & - & - & - & - \\
\hline Bond & Angles & Bond & Angles & Bond & Angles \\
\hline $\mathrm{O}(3)-\mathrm{N}(1)-\mathrm{O}(2)$ & $120.4(5)$ & $\mathrm{O}(10)-\mathrm{N}(4)-\mathrm{C}(8)$ & $118.5(6)$ & $\mathrm{C}(5)-\mathrm{C}(4)-\mathrm{N}(2)$ & $119.3(5)$ \\
\hline $\mathrm{O}(3)-\mathrm{N}(1)-\mathrm{C}(2)$ & $118.6(5)$ & $\mathrm{O}(9)-\mathrm{N}(4)-\mathrm{C}(8)$ & $118.5(6)$ & $\mathrm{C}(3)-\mathrm{C}(4)-\mathrm{N}(2)$ & $118.7(5)$ \\
\hline $\mathrm{O}(2)-\mathrm{N}(1)-\mathrm{C}(2)$ & $120.9(5)$ & $\mathrm{O}(11)-\mathrm{N}(5)-\mathrm{O}(12)$ & $124.0(5)$ & $\mathrm{C}(9)-\mathrm{C}(8)-\mathrm{N}(4)$ & $117.3(6)$ \\
\hline $\mathrm{O}(5)-\mathrm{N}(2)-\mathrm{O}(4)$ & $125.0(5)$ & $\mathrm{O}(11)-\mathrm{N}(5)-\mathrm{C}(10)$ & $117.8(5)$ & $\mathrm{C}(9)-\mathrm{C}(10)-\mathrm{N}(5)$ & $119.7(5)$ \\
\hline $\mathrm{O}(5)-\mathrm{N}(2)-\mathrm{C}(4)$ & $117.6(5)$ & $\mathrm{O}(12)-\mathrm{N}(5)-\mathrm{C}(10)$ & $118.2(5)$ & $\mathrm{C}(11)-\mathrm{C}(10)-\mathrm{N}(5)$ & $118.0(5)$ \\
\hline $\mathrm{O}(4)-\mathrm{N}(2)-\mathrm{C}(4)$ & $117.2(5)$ & $\mathrm{O}(14)-\mathrm{N}(6)-\mathrm{O}(13)$ & $120.9(7)$ & $\mathrm{C}(11)-\mathrm{C}(12)-\mathrm{N}(6)$ & $115.6(5)$ \\
\hline $\mathrm{O}(6)-\mathrm{N}(3)-\mathrm{O}(7)$ & $122.4(5)$ & $\mathrm{O}(14)-\mathrm{N}(6)-\mathrm{C}(12)$ & $120.6(6)$ & $\mathrm{O}(8)-\mathrm{C}(7)-\mathrm{C}(12)$ & $119.5(5)$ \\
\hline $\mathrm{O}(6)-\mathrm{N}(3)-\mathrm{C}(6)$ & $118.1(5)$ & $\mathrm{O}(13)-\mathrm{N}(6)-\mathrm{C}(12)$ & $118.4(5)$ & $C(12)-C(11)-C(10)$ & $117.9(5)$ \\
\hline $\mathrm{O}(7)-\mathrm{N}(3)-\mathrm{C}(6)$ & $119.5(4)$ & $\mathrm{O}(1)-\mathrm{C}(1)-\mathrm{C}(2)$ & $120.5(4)$ & $\mathrm{C}(7)-\mathrm{C}(12)-\mathrm{N}(6)$ & $122.2(5)$ \\
\hline $\mathrm{O}(10)-\mathrm{N}(4)-\mathrm{O}(9)$ & $122.9(6)$ & $\mathrm{O}(1)-\mathrm{C}(1)-\mathrm{C}(6)$ & $123.2(5)$ & $\mathrm{C}(7)-\mathrm{C}(8)-\mathrm{N}(4)$ & $120.7(5)$ \\
\hline $\mathrm{C}(5)-\mathrm{C}(6)-\mathrm{C}(1)$ & $122.5(5)$ & $\mathrm{O}(8)-\mathrm{C}(7)-\mathrm{C}(8)$ & $123.2(5)$ & $\mathrm{C}(11)-\mathrm{C}(12)-\mathrm{C}(7)$ & $122.2(5)$ \\
\hline $\mathrm{C}(5)-\mathrm{C}(6)-\mathrm{N}(3)$ & $117.9(5)$ & $\mathrm{C}(3)-\mathrm{C}(2)-\mathrm{N}(1)$ & $117.2(5)$ & $\mathrm{C}(1)-\mathrm{C}(6)-\mathrm{N}(3)$ & $119.6(5)$ \\
\hline $\mathrm{C}(1)-\mathrm{C}(2)-\mathrm{N}(1)$ & $121.1(5)$ & - & - & - & - \\
\hline
\end{tabular}

The structure was solved by using the program SHELXS-97 ${ }^{5}$ and Fourier difference techniques and refined by full-matrix least-squares method on $\mathrm{F}^{2}$ using SHELXL-97 ${ }^{6}$. Details of the data collection and refinements of title compound are given in Table-1. The non-hydrogen atoms were refined anisotropically. Hydrogen atoms were added theoretically.

\section{RESULTS AND DISCUSSION}

X-Ray crystallographic analysis revealed the crystal structure of 2,4,6-trinitrophenol and the structure is shown in Fig. 1. Selected bond distances and angles are listed in Table-
2. The crystal structure of the title compound is built up by only the $\mathrm{C}_{6} \mathrm{H}_{3} \mathrm{~N}_{3} \mathrm{O}_{7}$ molecules, in which all bond lengths are in normal ranges. There are two crystallographically independent but chemically identical molecules (A and B) in the asymmetric unit. In both molecules, the phenolic alcohol and nitro groups are essentially coplanar with the attached benzene ring. The two benzene rings form a dihedral angle of $c a .67 .49^{\circ}$.

In the crystal structure, there are four strong intramolecular $\mathrm{O}-\mathrm{H} \cdots \mathrm{O}$ and $\mathrm{O}-\mathrm{H} \cdots \mathrm{N}$ hydrogen bonds $(\mathrm{O}(1)-\mathrm{H}(1) \cdots \mathrm{O}(2), \mathrm{O}(1)-$ $\mathrm{H}(1) \cdots \mathrm{N}(1)$ in molecule $\mathrm{A}$ and $\mathrm{O}(8)-\mathrm{H}(8) \cdots \mathrm{O}(14), \mathrm{O}(8)-$ $\mathrm{H}(8) \cdots \mathrm{N}(6)$ in molecule B) between the phenolic hydroxyl

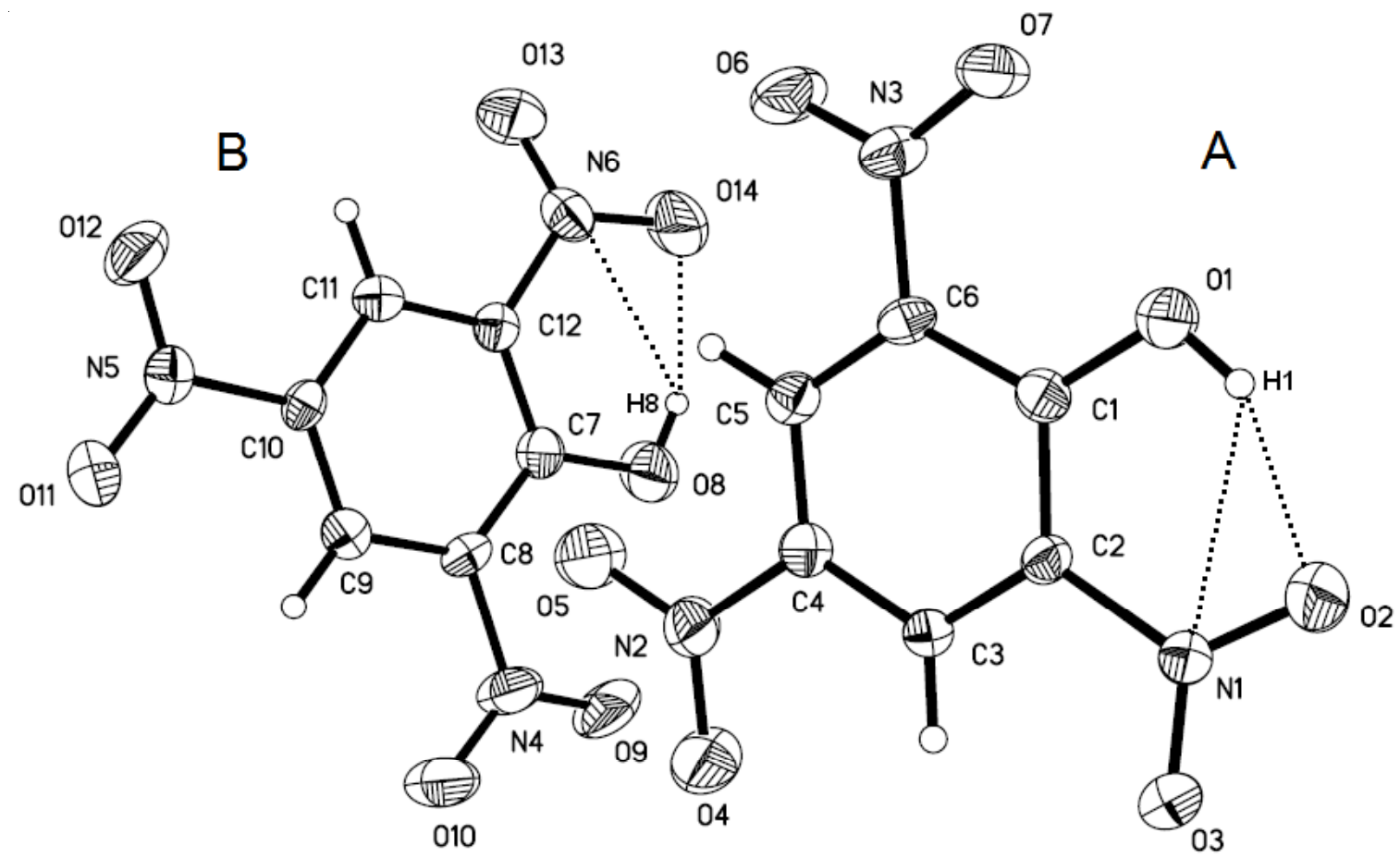

Fig. 1. Molecule structure of the title compound with atom numbering showing the intramolecular hydrogen bonds 


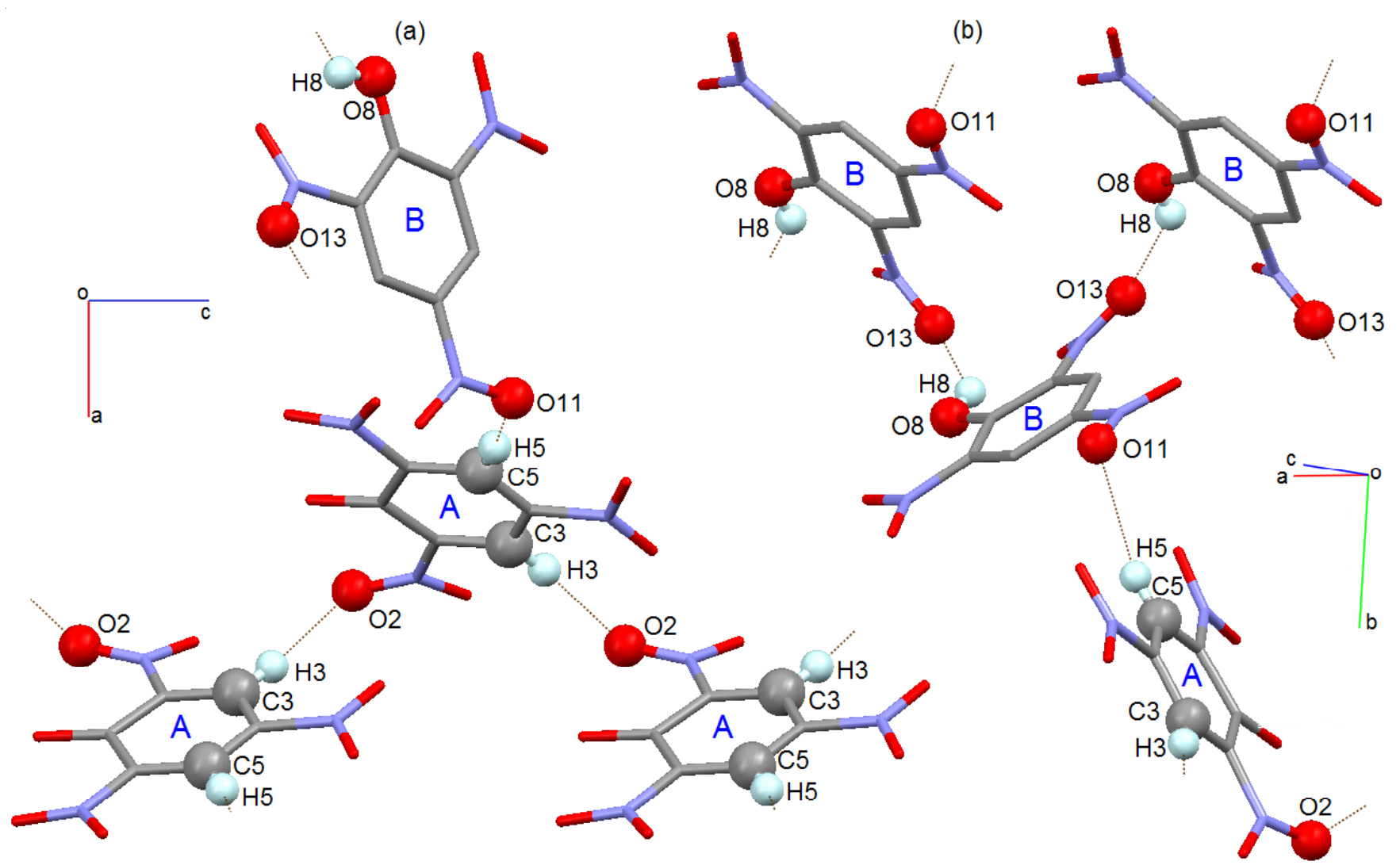

Fig. 2. View of the 2D supramolecular layer within the title compound (hydrogen atoms, except those forming hydrogen bonds, are omitted for clarity)

groups $(-\mathrm{OH})$ and the nitro oxygen $(\mathrm{O})$ and nitro nitrogen $(\mathrm{N})$ atoms (Table-3), which generate six-membered $\mathrm{S}(6)$ ring motifs (Fig. 1). In addition, every molecule A links two other molecules A via intermolecular $\mathrm{C}(3)-\mathrm{H}(3) \cdots \mathrm{O}(2)$ hydrogenbonding and one molecules B via intermolecular $\mathrm{C}(5)$ $\mathrm{H}(5) \cdots \mathrm{O}(11)$ hydrogen-bonding interactions into an infinite 2D-layer supramolecular structure on the ac crystallographic plane (Fig. 2a). Whereas, every molecule B links two other molecules B via intermolecular $\mathrm{O}(8)-\mathrm{H}(8) \cdots \mathrm{O}(13)$ hydrogenbonding and one molecules A via intermolecular $\mathrm{C}(5)$ $\mathrm{H}(5) \cdots \mathrm{O}(11)$ hydrogen-bonding interactions into the other infinite 2D-layer supramolecular structure on the ab crystallographic plane (Fig. 2b). Thus, the crystal packing of the compound shows a self-assembling infinite three-dimensional supramolecular network structure.

\begin{tabular}{lcccc}
\multicolumn{5}{c}{ TABLE-3 } \\
HYDROGEN-BONDING DATA $\left[\AA{ }^{\circ}{ }^{\circ}\right]$ \\
\hline \multicolumn{1}{c}{$\mathrm{D}-\mathrm{H} \cdots \mathrm{A}$} & $\mathrm{d}(\mathrm{D}-\mathrm{H})$ & $\mathrm{d}(\mathrm{H} \cdots \mathrm{A})$ & $\mathrm{d}(\mathrm{D} \cdots \mathrm{A})$ & $\angle \mathrm{D}-\mathrm{H} \cdots \mathrm{A}$ \\
\hline $\mathrm{O}(1)-\mathrm{H}(1) \cdots \mathrm{O}(2)$ & 0.820 & 1.88 & $2.599(6)$ & 146 \\
$\mathrm{O}(1)-\mathrm{H}(1) \cdots \mathrm{N}(1)$ & 0.820 & 2.346 & $2.839(2)$ & 118 \\
$\mathrm{O}(8)-\mathrm{H}(8) \cdots \mathrm{O}(14)$ & 0.820 & 1.925 & $2.622(9)$ & 142 \\
$\mathrm{O}(8)-\mathrm{H}(8) \cdots \mathrm{N}(6)$ & 0.820 & 2.370 & $2.841(3)$ & 118 \\
$\mathrm{O}(8)-\mathrm{H}(8) \cdots \mathrm{O}(13)$ & 0.820 & 2.601 & $3.252(3)$ & 138 \\
$\mathrm{C}(3)-\mathrm{H}(3) \cdots \mathrm{O}(2)$ & 0.930 & 2.450 & $3.373(7)$ & 173 \\
$\mathrm{C}(5)-\mathrm{H}(5) \cdots \mathrm{O}(11)$ & 0.930 & 2.628 & $3.540(7)$ & 167 \\
\hline
\end{tabular}

\section{ACKNOWLEDGEMENTS}

The authors thank the Young Scholars Science Foundation of Lanzhou Jiaotong University (2011007) for financial support of this work.

\section{REFERENCES}

1. R. Narayanan, P. Tiwari, D. Inoa and B.T. Ashok, Life Sci., 77, 2312 (2005).

2. S. Saito and Y. Koizumi, Tetrahedron Lett., 46, 4715 (2005).

3. P. Srinivasan, M. Gunasekaran, T. Kanagasekaran, R. Gopalakrishnan and P. Ramasamy, J. Crystal Growth, 289, 639 (2006).

4. C. Muthamizhchelvan, K. Saminathan, J. Fraanje, R. Peschar and K. Sivakumar, X-Ray Struct. Online, 21, 59 (2005).

5. G.M. Sheldrick, SHELXS97, Program for Crystal Structure Determination, University of Göttingen, Germany (1996).

6. G.M. Sheldrick, Acta Cryst., A64, 112 (2008). 